\title{
Relationship between the Spectral Power Density of Sleep Electroencephalography and Psychiatric Symptoms in Patients with Breathing-related Sleep Disorder
}

\author{
Jae Myeong Kang ${ }^{1}$, Seo-Eun Cho ${ }^{1}$, Gun Bae Lee ${ }^{2}$, Seong-Jin Cho ${ }^{1}$, Kee Hyung Park ${ }^{3}$, Seon Tae Kim ${ }^{4}$, \\ Seung-Gul Kang ${ }^{1}$ \\ ${ }^{1}$ Department of Psychiatry, Gachon University Gil Medical Center, Gachon University College of Medicine, ${ }^{2}$ Gachon Sleep Medicine Center, \\ Gachon University Gil Medical Center, ${ }^{3}$ Department of Neurology, ${ }^{4}$ Department of Otolaryngology, Gachon University Gil Medical Center, \\ Gachon University College of Medicine, Incheon, Korea
}

\begin{abstract}
Objective: Patients with breathing-related sleep disorder (BRSD) often complain of psychiatric symptoms such as depression in addition to snoring, excessive sleepiness, and disturbed sleep. However, the relationship between psychiatric symptoms and severity of sleep apnea in BRSD is controversial. We conducted this study to investigate the relationship between psychiatric symptoms and sleep electroencephalography (EEG) findings in BRSD patients using spectral analysis. Methods: All participants underwent polysomnography and evaluation using Symptom Checklist-90-Revised (SCL-90-R) scale. We analyzed the absolute spectral power density values of standard EEG frequency bands in the participants $(n=169$ ) with BRSD during the non-rapid eye movement (NREM) sleep period. We performed correlation analysis between the domain scores of SCL-90-R scale and the absolute values of the EEG frequency bands.

Results: Significant positive correlation was observed between the absolute spectral power density values in the slow oscillation band and the degree of paranoid ideation $(r=0.226, p=0.028)$ and depression $(r=0.216, p=0.044)$ in SCL-90-R. The multiple linear regression model showed that higher paranoid ideation domain score $(B=0.007$, $p=0.020)$, younger age $(B=-0.011, p<0.001)$, and female sex $(B=0.213, p=0.004)$ were associated with higher slow oscillation power during NREM sleep.

Conclusion: The results of the present study suggested a relationship between sleep EEG and psychiatric symptoms in patients with BRSD. This relationship needs to be validated with further studies.
\end{abstract}

KEY WORDS: Breathing-related sleep disorder; Sleep electroencephalography; Spectral analysis; Symptom Checklist90-Revised; Polysomnography.

\section{INTRODUCTION}

Breathing-related sleep disorder (BRSD) is a common sleep disorder characterized by snoring, recurrent episodes of partial or complete collapse of the upper airway, and witnessed apnea during sleep. BRSD can be clinically diagnosed using the Diagnostic and Statistical Manual of Mental Disorders (fourth edition, text revision) (DSM-IV-TR)

Received: July 20, 2020 / Revised: August 28, 2020

Accepted: September 5, 2020

Address for correspondence: Seung-Gul Kang

Department of Psychiatry, Gachon University Gil Medical Center,

Gachon University College of Medicine, 21 Namdong-daero

774beon-gil, Namdong-gu, Incheon 21565, Korea

E-mail: kangsg@gachon.ac.kr

ORCID: https://orcid.org/0000-0003-4933-0433
[1]. However, polysomnography (PSG) is required for accurate diagnosis. BRSD can be classified into simple snoring (SS), upper airway resistance syndrome (UARS), and obstructive sleep apnea (OSA) according to the apnea-hypopnea index (AHI) scores [2]. OSA is diagnosed when apnea or hypopnea occurs five or more times per hour $(\mathrm{AHI} \geq 5$ ) [3]. In the BRSD spectrum, $\mathrm{SS}$ is defined as $\mathrm{AHI}<5$ and UARS is defined as AHI $<5$ and respiratory disturbance index $>5$.

Common symptoms of BRSD such as snoring, fatigue, and sleepiness can affect daytime functioning. Moreover, psychiatric symptoms such as depression, anxiety, and irritability are frequently reported [4]. Previous studies have explored the relationship between BRSD and psychiatric

(ㄷ) This is an Open-Access article distributed under the terms of the Creative Commons Attribution Non-Commercial License (http://creativecommons.org/licenses/by-nc/4.0) which permits unrestricted non-commercial use, distribution, and reproduction in any medium, provided the original work is properly cited. 
symptoms. OSA could induce depression-like symptoms such as hypersomnolence, fatigue, lack of energy, and difficulty in concentration [4]. The reciprocal effects of BRSD and depression on each other were reported in a large cross-sectional study [5]. The risk of major depressive disorder in patients with OSA compared to normal individuals was 5.2 times and 2.4 times for the female and the male participants, respectively [6]. Depression and anxiety were also found in snorers [7]. However, previous studies have shown inconsistent results regarding the correlation between severity of BRSD (measured with AHI) and severity of psychiatric symptoms such as depression and anxiety. In a previous study, patients with OSA showed more intense somatic and depressive symptoms compared to patients with SS $[4,8]$. In contrast, other studies showed no significant differences in depression and anxiety between patients with OSA and patients with SS $[7,9,10]$. We found more severe psychiatric symptoms in the SS group when compared with the OSA group in our previous study [11].

The relationship between severity of BRSD and psychiatric symptoms is still unclear. It is difficult to explain the psychological symptoms of BRSD using the macrostructure of PSG. Many psychiatric disorders including psychotic disorder and depressive disorder are associated with symptoms of sleep disturbance from the early phases of these disorders [12]. Sleep abnormalities have been associated with severity of psychiatric symptoms [13]. Sleep disturbances often precede psychotic or depressive episodes $[14,15]$. Healthy individuals with a high genetic load for psychiatric disorders showed sleep pattern similar to the sleep pattern in depression observed on PSG. This result suggested the possibility of sleep pattern being a trait marker for vulnerability to psychiatric disorders [16]. In addition, previous studies have analyzed the relationship of sleep electroencephalography (EEG) with mental disorders, psychiatric symptoms, and treatment responses to medication [17-20]. Fast sleep spindle reduction on sleep EEG and its association with cognitive impairment was observed in schizophrenia [17]. Depression is characterized by an overall decrease in the slow-wave activities, which is related to elevated anxiety and depressed mood the following morning [19]. Therefore, we can postulate that the psychiatric symptoms in BRSD may be related to sleep EEG findings.

Researchers have previously attempted to investigate the microstructure of PSG-derived sleep as well as waking quantitative EEG (qEEG) in BRSD [21-25]. The sigma (13$15 \mathrm{~Hz}$ ) power of sleep EEG predicted next-day sleepiness, as measured by the multiple sleep latency test [21] Low-resolution electromagnetic tomography and sleep and wake qEEG analyses showed decreased alpha activity in the right posterior cingulate gyrus in patients with severe OSA than in patients having mild OSA [24]. Despite the results of many spectral analyses of sleep and wake qEEG in OSA or BRSD patients, no study has analyzed the association between psychiatric symptoms and spectral power densities of sleep EEG in patients with BRSD, to the best of our knowledge.

Our goal was to study the relationship between the spectral power density of sleep EEG and the degree of psychiatric symptoms in BRSD patients. If a significant correlation between the power spectra in a specific sleep EEG band and psychiatric symptoms could be found, we also aimed to find whether severity of BRSD and demographic characteristics in addition to psychiatric symptoms could predict EEG power of the specific spectrum.

\section{METHODS}

\section{Participants}

We recruited adults (aged 18-65 years) with BRSD from the Gachon Sleep Medicine Center in Gil Medical Center from March 2012 to February 2016. Among the 205 participants recruited through screening scales and structured clinical interviews, 169 were included in the final analyses. All participants had symptoms of BRSD such as frequent snoring, daytime sleepiness, and witnessed apnea. All participants met the BRSD diagnostic criteria according to DSM-IV-TR [1]. Diagnoses of BRSD of the participants were confirmed by interviews with boardcertified medical doctors in the departments of otolaryngology, psychiatry, and neurology who had experience of over 5 years in sleep medicine and BRSD.

We excluded participants with following conditions: 1) current or past history of major psychiatric disorders; 2) comorbidities of severe medical conditions; 3) previous diagnosis with OSA; 4) history of uvulopalatopharyngoplasty; and 5) other suspected major sleep disorders such as rapid eye movement (REM) sleep behavior disorder, restless legs syndrome, circadian rhythm sleep disorder, or narcolepsy. 
All participants provided written informed consent and the Institutional Review Board of Gil Medical Center approved the study protocols (GIRBA2764-2012). We followed the relevant guidelines and regulations for all experiments throughout the study period.

\section{Questionnaire for the Evaluation of the Psychiatric Symptoms}

All participants completed a questionnaire about psychiatric symptoms, namely the Symptom Checklist-90Revised (SCL-90-R) scale comprising of 90 items [26]. SCL-90-R is a self-report inventory designed to evaluate patterns of psychological symptoms in psychiatric and medical patients. The results are shown as nine domain scores of primary symptom dimensions including somatization, obsessive-compulsive, depression, interpersonal sensitivity, anxiety, hostility, phobic anxiety, paranoia, and psychotic symptoms and three global indices, namely the global severity index, the positive symptoms total, and the positive symptoms distress index. Each question is scored based on a 5-point Likert scale from 0 (none) to 4 (extreme). We used the Korean version of SCL-90-R validated in Korean population [27].

\section{Polysomnography}

All participants underwent laboratory night PSG in accordance with the recommendations of American Academy of Sleep Medicine (AASM) [28]. Standard PSG recordings were conducted using the COMET system (Grass-Telefactor Corporation, West Warwick, RI, USA) with six EEG leads (F3, F4, C3, C4, O1, and O2), two electrooculogram channels (E1-M2 and E2-M2), three electromyography channels, and an electrocardiography channel. The PSG parameters were scored based on the criteria in the AASM manual [28]. According to the recommended rules of the AASM manual, we defined hypopnea as $\geq 30 \%$ reduction in nasal pressure signal excursions from baseline lasting $\geq 10$ seconds associated with $\geq 4 \%$ desaturation from the pre-event baseline [28]. All PSG recordings were conducted by experienced PSG technologists who underwent and completed the interscorer reliability program (http://www.aasmnet.org/isr/) using the AASM sleep scoring criteria [28]. A board-certified neurologist and sleep specialist medical doctor (K.H.P.) confirmed all PSG data.

\section{Spectral Analysis}

We computed power spectra for each EEG frequency band, namely slow oscillation band $(0.5-1 \mathrm{~Hz})$, delta $(1-$ $4 \mathrm{~Hz})$, theta $(4-8 \mathrm{~Hz})$, alpha $(8-12 \mathrm{~Hz})$, sigma $(12-15$ $\mathrm{Hz})$, and beta $(15-20 \mathrm{~Hz})$. In the present analysis, we used the data derived from the average non-REM (NREM) central EEG electrode ([C3/A2 + C4/A1] / 2).

We used SpectralTrainFig (National Sleep Research Resource, Boston, MI, USA) for the spectral power analysis (https://github.com/nsrr/SpectralTrainFig) [29,30]. This open-source Matlab graphic interface is designed for the spectral analysis of sleep EEG in PSG. It automatically detects and deletes epochs with artifacts and yields summary figures for visual adjudication [31,32]. According to Welch's method, it calculates the spectral power density using ten overlapping 4-second sub-epochs for each 30 -second epoch with a 50\% tapered cosine window. It removes the artifact resulting from electrocardiogram interference using a template subtraction method [33]. Manual visual adjudication and exclusion of spectral data with significant artifacts were conducted by a researcher who was blinded to the information about the participants.

\section{Statistical Analysis}

Descriptive statistics were used for the characterization of the participants and Pearson correlation analysis was used to find the correlation between the SCL-90-R score and the absolute spectral EEG power in the participants. For the SCL-90-R variables that deviated from normal distribution, log transformation was conducted before the correlation analysis. Significance was defined as $p<$ 0.050 after Bonferroni correction calculated by uncorrected $p$ value $\times 9$, as 9 was the number of domains on the SCL-90-R scale. The linear multiple regression model with stepwise method was used to find the best predictive factor for the specific EEG band significantly correlated with SCL-90-R. In the regression model, the independent variables included demographic information (age and sex), severity of BRSD (AHI), and psychiatric symptom domain scores (SCL-90-R) with significant correlation. IBM SPSS Statistics for Windows ver. 23 (IBM Corp., Armonk, NY, USA) was used for all analyses with statistical significance set at $p<0.050$ (two-tailed). 


\section{RESULTS}

\section{Demographic and PSG Characteristics of the Participants}

Demographic and PSG characteristics of the partic-

Table 1. Demographic data, polysomnographic findings, psychiatric symptoms, and spectral power density of participants $(n=169)$

\begin{tabular}{|c|c|}
\hline Variable & Statistics \\
\hline \multicolumn{2}{|l|}{ Demographics } \\
\hline Age $(y r)$ & $45.7 \pm 11.3$ \\
\hline Sex (male) & $143(84.6)$ \\
\hline Body mass index $\left(\mathrm{kg} / \mathrm{m}^{2}\right)$ & $25.7 \pm 3.5$ \\
\hline \multicolumn{2}{|l|}{ Polysomnographic data } \\
\hline \multicolumn{2}{|l|}{ Sleep and wake time } \\
\hline Time in bed (min) & $412.1 \pm 28.9$ \\
\hline Total sleep time (min) & $338.0 \pm 56.0$ \\
\hline Sleep latency (min) & $13.5 \pm 21.9$ \\
\hline Sleep efficiency (\%) & $82.1 \pm 13.6$ \\
\hline WASO (min) & $59.8 \pm 48.8$ \\
\hline REM sleep latency (min) & $124.4 \pm 62.3$ \\
\hline \multicolumn{2}{|l|}{ Sleep stage $(\%)$} \\
\hline N1 & $27.4 \pm 15.8$ \\
\hline N2 & $53.0 \pm 14.4$ \\
\hline N3 & $3.4 \pm 5.6$ \\
\hline $\mathrm{R}$ & $15.9 \pm 7.1$ \\
\hline \multicolumn{2}{|l|}{ Respiration } \\
\hline AHI (events per hour) & $24.5 \pm 22.6$ \\
\hline Oxygen desaturation index & $20.6 \pm 20.2$ \\
\hline Lowest $\mathrm{O}_{2}$ saturation & $80.9 \pm 8.8$ \\
\hline Arousal index & $31.1 \pm 17.9$ \\
\hline \multicolumn{2}{|l|}{ SCL-90-R scores } \\
\hline Somatization & $50.8 \pm 11.9$ \\
\hline Obsessive-compulsive & $44.5 \pm 9.3$ \\
\hline Interpersonal sensitivity & $41.0 \pm 9.8$ \\
\hline Depression & $45.4 \pm 11.0$ \\
\hline Anxiety & $44.1 \pm 9.1$ \\
\hline Hostility & $44.2 \pm 7.7$ \\
\hline Phobic anxiety & $43.8 \pm 7.4$ \\
\hline Paranoid ideation & $42.5 \pm 9.0$ \\
\hline Psychoticism & $44.4 \pm 7.7$ \\
\hline Global severity index & $32.5 \pm 0.5$ \\
\hline Positive symptom total & $42.6 \pm 11.3$ \\
\hline Positive symptom distress index & $43.8 \pm 12.6$ \\
\hline \multicolumn{2}{|c|}{ Spectral power density ${ }^{a}$ during NREM sleep } \\
\hline Slow oscillation $(0.5-1 \mathrm{~Hz})$ & $2.07 \pm 0.36$ \\
\hline Delta $(1-4 \mathrm{~Hz})$ & $1.19 \pm 0.19$ \\
\hline Theta $(4-8 \mathrm{~Hz})$ & $0.63 \pm 0.22$ \\
\hline Alpha $(8-12 \mathrm{~Hz})$ & $0.34 \pm 0.24$ \\
\hline Sigma $(12-15 \mathrm{~Hz})$ & $0.12 \pm 0.22$ \\
\hline Beta $(15-20 \mathrm{~Hz})$ & $-0.38 \pm 0.23$ \\
\hline
\end{tabular}

Values are presented as mean \pm standard deviation or number (\%). WASO, wake time after sleep onset; REM, rapid eye movement; N1, non-REM (NREM) stage 1; N2, NREM stage 2; N3, NREM stage 3; R, REM stage; AHI, apnea hypopnea index; SCL-90-R, Symptom Checklist90-Revised NREM, non-rapid eye movement.

${ }^{\mathrm{a} L o g-t r a n s f o r m e d ~ s p e c t r a l ~ p o w e r ~ d e n s i t y ~}(\log 10 \mu \mathrm{V} 2)$. ipants are presented in Table 1. The average age was 45.7 years and male subjects constituted $84.6 \%$ of the total sample (total 169 participants). The description of the PSG data is shown in Table 1. The average total sleep time was 338.0 minutes and sleep efficiency was $82.1 \%$; average AHI was 24.5 per hour and arousal index was 31.1 per hour.

\section{SCL-90-R Scores and Absolute Spectral EEG Power of Participants}

Table 1 presents the description of the domain scores for nine primary symptom dimensions and three global indices of the SCL-90-R scale. The mean domain scores of the nine dimensions ranged from 41.0 to 50.8 .

The absolute spectral EEG power of the participants is presented in Table 1. The log-transformed absolute spectral power densities were $2.07 \pm 0.36,1.19 \pm 0.19,0.63 \pm$ $0.22,0.34 \pm 0.24,0.12 \pm 0.22$, and $-0.38 \pm 0.23$ for slow oscillation band, delta, theta, alpha, sigma, and beta band, respectively.

\section{Correlation Analysis between Absolute Spectral Power Density and Domain Scores of SCL-90-R}

Table 2 presents the results of the correlation analyses between absolute spectral power density and domain scores of the nine primary symptom dimensions in the SCL-90-R scale. Log transformation for domain scores of SCL-90-R was performed before Pearson correlation analysis when the variables deviated from normal distribution. Slow oscillation during NREM sleep significantly correlated with paranoid ideation score $(r=0.226, p=0.003$, corrected $p=0.028$; Table 2$)$ and with depression score $(r=$ $0.216, p=0.005$, corrected $p=0.044$; Table 2).

\section{Multiple Linear Regression Models for Slow Oscillation Power during NREM Sleep}

The results of the multiple linear regression models using slow oscillation power that significantly correlated with paranoid ideation and depression scores of SCL-90-R as the dependent variable are presented in Table 3. In the multiple regression model, higher slow oscillation power was found to be predicted by higher paranoid ideation domain score $(\mathrm{B}=0.007, p=0.020)$, younger age $(\mathrm{B}=-0.011$, $p<0.001)$, and female sex $(\mathrm{B}=0.213, p=0.004)$. AHI $(p=$ $0.13)$ and depression domain score $(p=0.77)$ were excluded from the stepwise multiple linear regression model. 
Table 2. Correlation analysis between SCL-90-R domain scores and absolute spectral power densities of sleep EEG during NREM sleep

\begin{tabular}{|c|c|c|c|c|c|c|c|c|c|}
\hline \multirow{2}{*}{ Variable } & \multicolumn{3}{|c|}{ Slow oscillation } & \multicolumn{3}{|c|}{ Delta } & \multicolumn{3}{|c|}{ Theta } \\
\hline & $r^{a}$ & $p$ value & $p$ corr & $r^{\mathrm{a}}$ & $p$ value & $p$ corr & $r^{\mathrm{a}}$ & $p$ value & $p$ corr \\
\hline SOM & -0.023 & 0.77 & $>0.99$ & 0.119 & 0.12 & $>0.99$ & 0.135 & 0.080 & 0.72 \\
\hline $\mathrm{O}-\mathrm{C}$ & 0.203 & 0.008 & 0.072 & 0.144 & 0.061 & 0.54 & 0.096 & 0.21 & $>0.99$ \\
\hline I-S & 0.204 & 0.008 & 0.072 & 0.088 & 0.25 & $>0.99$ & 0.032 & 0.67 & $>0.99$ \\
\hline DEP & 0.216 & 0.005 & $0.044^{b}$ & 0.129 & 0.095 & 0.85 & 0.086 & 0.26 & $>0.99$ \\
\hline ANX & 0.160 & 0.038 & 0.34 & 0.084 & 0.27 & $>0.99$ & 0.082 & 0.29 & $>0.99$ \\
\hline HOS & 0.180 & 0.019 & 0.17 & 0.130 & 0.093 & 0.83 & 0.115 & 0.13 & $>0.99$ \\
\hline $\mathrm{PHOB}$ & 0.173 & 0.025 & 0.22 & 0.075 & 0.33 & $>0.99$ & 0.098 & 0.20 & $>0.99$ \\
\hline PAR & 0.226 & 0.003 & $0.028^{\mathrm{b}}$ & 0.061 & 0.43 & $>0.99$ & 0.021 & 0.78 & $>0.99$ \\
\hline \multirow[t]{3}{*}{ PSY } & 0.099 & 0.20 & $>0.99$ & 0.078 & 0.31 & $>0.99$ & 0.080 & 0.30 & $>0.99$ \\
\hline & \multicolumn{3}{|c|}{ Alpha } & \multicolumn{3}{|c|}{ Sigma } & \multicolumn{3}{|c|}{ Beta } \\
\hline & $r^{\mathrm{a}}$ & $p$ value & $p$ corr & $r^{2}$ & $p$ value & $p$ corr & $r^{\mathrm{a}}$ & $p$ value & $p$ corr \\
\hline SOM & 0.146 & 0.058 & 0.52 & 0.167 & 0.030 & 0.27 & 0.111 & 0.15 & $>0.99$ \\
\hline $\mathrm{O}-\mathrm{C}$ & 0.111 & 0.15 & $>0.99$ & 0.130 & 0.093 & 0.83 & 0.059 & 0.44 & $>0.99$ \\
\hline I-S & 0.042 & 0.59 & $>0.99$ & 0.092 & 0.23 & $>0.99$ & 0.041 & 0.59 & $>0.99$ \\
\hline DEP & 0.081 & 0.29 & $>0.99$ & 0.088 & 0.25 & $>0.99$ & 0.054 & 0.48 & $>0.99$ \\
\hline ANX & 0.099 & 0.20 & $>0.99$ & 0.126 & 0.10 & 0.92 & 0.080 & 0.30 & $>0.99$ \\
\hline HOS & 0.077 & 0.32 & $>0.99$ & 0.100 & 0.19 & $>0.99$ & 0.067 & 0.38 & $>0.99$ \\
\hline $\mathrm{PHOB}$ & 0.065 & 0.39 & $>0.99$ & 0.086 & 0.26 & $>0.99$ & 0.084 & 0.27 & $>0.99$ \\
\hline PAR & -0.006 & 0.94 & $>0.99$ & 0.026 & 0.73 & $>0.99$ & 0.007 & 0.93 & $>0.99$ \\
\hline PSY & 0.081 & 0.29 & $>0.99$ & 0.130 & 0.092 & 0.82 & 0.082 & 0.28 & $>0.99$ \\
\hline
\end{tabular}

SCL-90-R, Symptom Checklist-90-Revised; EEG, electroencephalography; NREM, non-rapid eye movement $r$, Pearson's $r p$ corr, $p$ value after Bonferroni correction (uncorrected $p$ value $\times 9$ ) for correction of multiple comparisons; SOM, somatization; O-C, obsessive-compulsive; I-S, interpersonal sensitivity; DEP, depression; ANX, anxiety; HOS, hostility; PHOB, phobic anxiety; PAR, paranoid ideation; PSY, psychoticism. a Log transformation for domain scores of SCL-90-R was performed before Pearson correlation analysis when the variables deviated from normal distribution. ${ }^{\mathrm{b}}$ Significant after Bonferroni correction $(p<0.050)$.

Table 3. Multiple linear regression models for sleep oscillation power during NREM sleep

\begin{tabular}{|c|c|c|c|c|c|}
\hline \multirow{2}{*}{$\begin{array}{l}\text { Independent } \\
\text { variable }\end{array}$} & \multicolumn{5}{|c|}{ Slow oscillation } \\
\hline & B & $\begin{array}{c}\text { Standard } \\
\text { error }\end{array}$ & $\beta$ & $p$ value & $\mathrm{R}^{2}$ \\
\hline Age & -0.011 & 0.002 & -0.356 & $<0.001^{\mathrm{a}}$ & 0.20 \\
\hline Sex & 0.213 & 0.072 & 0.213 & $0.004^{\mathrm{a}}$ & \\
\hline PAR of SCL-90-R & 0.007 & 0.003 & 0.167 & $0.020^{\mathrm{a}}$ & \\
\hline \multicolumn{6}{|l|}{ Excluded variable } \\
\hline $\mathrm{AHI}$ & & & & 0.13 & \\
\hline DEP of SCL-90-R & & & & 0.77 & \\
\hline
\end{tabular}

Dependent variable: slow oscillation power during NREM sleep. Multiple regression model: independent variables included age, sex, PAR domain score of SCL-90-R, DEP domain score of SCL-90-R, and AHI. $\mathrm{AHI}$ and DEP scores were excluded from the stepwise multiple linear regression model.

NREM, non-rapid eye movement PAR, paranoid ideation; SCL-90-R, Symptom Checklist-90-Revised; AHI, apnea-hypopnea index; DEP, depression.

a $p<0.050$.

\section{DISCUSSION}

We conducted spectral power analysis of PSG-derived sleep EEG in patients with BRSD and analyzed the correlation between spectral power density and domain scores of SCL-90-R. The significant finding of the present study was the positive correlation between slow oscillation during NREM sleep and paranoid ideation and depression domain scores of SCL-90-R. In addition, multiple linear regression analysis showed that younger age, female sex, and paranoid ideation domain score had a significant effect on increased slow oscillation during NREM sleep.

The domain scores of SCL-90-R in our study were mostly between 40 and 50 . Only the domain score for somatization was over 50 points, which is the cut-off score of abnormality [27]. The domain scores were similar to those reported in a previous Korean study that compared the SCL-90-R scores between tinnitus patients without psychiatric illness and control subjects [34].

In the present study, slow oscillation during NREM 
sleep was significantly correlated with paranoid ideation and depressive symptoms. Slow oscillation has not been sufficiently studied in previous human studies. Hence, its clinical significance has not been established. Slow oscillations $(<1 \mathrm{~Hz})$ in the NREM sleep EEG originate from the cortical neurons of the brain and are known to alternate between a depolarized up-state and a hyperpolarized down-state in association with slow membrane potential fluctuation [35]. A previous study has reported slow oscillation increases while sleeping after prolonged wakefulness [35]. NREM sleep in patients with post-traumatic stress disorder showed decreased slow oscillation power and increased higher frequency activity compared to that in control subjects. On the contrary, the slow oscillation power during REM sleep increased in the occipital area [36]. In addition, a study comparing sleep EEG among subjects with primary insomnia, chronic fatigue syndrome, and normal controls showed consistently lower proportion of slow oscillation power during slow wave sleep in primary insomnia and chronic fatigue syndrome, although the proportion of slow wave sleep duration varied among the groups [37]. A large-scale computer model study of the thalamocortical system during sleep found that a decrease in the cortical synaptic strength could explain the decrease in the slow-wave activity including slow oscillation during sleep [38]. In the present study, as the domain scores of paranoid ideation and depression increased, the spectral power density in slow oscillation increased during NREM sleep and paranoid ideation significantly predicted slow oscillation in the regression analyses. Little is known about the relationship between paranoia and BRSD. A large previous cohort study has reported increased prevalence of psychotic disorder in patients with OSA [39], and case studies have found increased psychotic depression [40], hallucination, and delirium in OSA patients [41], and improved psychotic symptoms after treating sleep apnea [42]. Association between psychotic symptoms and sleep EEG has not been investigated in BRSD patients; however, low delta [43] and high alpha power [44] in schizophrenia patients have been reported. The association between psychiatric symptoms and spectral power density in BRSD has not been investigated, and our results seem inconsistent with the results of previous studies in schizophrenia patients. This inconsistency may be attributed to the exclusion of subjects with major psychiatric disorders in our study. In addition, we believe that slow oscillation increased as a compensatory mechanism to overcome sleep insufficiency due to increased psychiatric symptoms. However, it is difficult to draw inferences regarding the cause-effect relationship due to the limitations posed by the cross-sectional nature of this study.

Results of the regression analysis revealed that slow oscillation decreased with age and increased in female sex. Previous studies have reported that the number and the amplitude of slow oscillation decreases with increasing age [45-47]. In addition, it seems that slow oscillation and sleep spindle generation and their coupling is also impaired in old age [48]. This is presumably a secondary result of normal age-related changes in the brain structure $[46,49]$. Young adult female have shown greater amount of slow wave sleep during NREM sleep than male in a previous study [50]. Older female showed more amount of slow wave sleep than older male of the same age during their sleep [51,52], although females usually complain of insomnia more often and no interaction effect of age and sex on slow wave sleep emerged in another sleep qEEG study [53]. The relationship among slow oscillation, age, and sex observed in the regression analysis of the present study is consistent with the results of the previous studies.

In the present study, AHI was not associated with slow oscillation during NREM sleep in BRSD patients. This result is in accordance with the results of previous studies showing weak relationship between $\mathrm{AHI}$ and symptoms of BRSD such as daytime sleepiness and sleep perception [54-56]. It has also been reported that psychiatric symptoms such as depression and anxiety were elevated in SS patients when compared with those in OSA patients [7]. We have also reported comparable results in previous studies and found no association among psychiatric symptoms, quality of life, and AHI in BRSD [11,57]. Thus, the results of the present result may suggest that the objective severity of BRSD is not associated even with the microstructural characteristics of BRSD patients.

To the best of our knowledge, there have been no spectral analysis studies regarding the relationship between psychiatric symptoms and sleep EEG in patients with BRSD. In a previous spectral analysis study of BRSD, sleep qEEG was used to investigate the EEG changes during transient hypoxia induced by apnea or hypopnea [58]. In another study that investigated EEG during periods of sleep apnea in REM and NREM sleep, the delta activity in- 
creased progressively and was generalized over the posterior frontal, central, and parietal regions during NREM apneas [59]. A previous study has found EEG slowing in REM sleep during apnea over the frontal, central, and parietal regions in OSA patients when compared with normal controls [60]. It was also reported that decreased brain activity was associated with apnea events [61]. We found that an increase in slow oscillation was associated with paranoid ideation of the BRSD patients. This finding may suggest the compensatory role of slow oscillation activity in psychiatric symptoms of BRSD.

This study has several limitations. The cross-sectional design might limit conclusions regarding a causal relationship. We suggest future studies with a longitudinal design to investigate the effect of psychiatric symptoms on the microstructure of BRSD patients' sleep. Modest effect sizes $(r=0.226$ for correlation and $\beta=0.167$ for regression analyses) and relatively low values of paranoid ideation are other limitations of the results of the present study that might limit the explanatory power. Future studies with a large sample size and thorough evaluation of psychiatric symptoms, which would increase the power of the investigation, will be necessary.

This is the first study to investigate the relationship between psychiatric symptoms and sleep EEG in BRSD patients. We found a significant relationship between slow oscillation and psychiatric symptoms such as paranoid ideation and depression during NREM sleep. Factors associated with increased slow oscillation were younger age, female sex, and greater paranoid ideation, suggesting the compensatory role of slow oscillation in psychiatric symptoms of BRSD. Further prospective studies are needed in future to elucidate the relationship among varied phenotypes of BRSD, microstructure of PSG, and the mechanism of sleep EEG activation.

\section{Acknowledgments}

This work was supported by a grant from the Korea Health Technology R\&D Project through the Korea Health Industry Development Institute, funded by the Ministry of Health and Welfare, Republic of Korea (grant number: HI17C2665); and a grant from the National Research Foundation of Korea (NRF) grant funded by the Korea government (MSIT) (grant number: NRF-2020R1A2C1007527).

We thank Sara Mariani for the spectral analysis and we also thank Dr. Dennis Dean, PhD and National Sleep
Research Resource for creating the SpectralTrainFig.

\section{- Conflicts of Interest}

No potential conflict of interest relevant to this article was reported.

\section{Author Contributions}

Conceptualization: Seung-Gul Kang. Data curation: Seon Tae Kim, Gun Bae Lee, Kee Hyung Park, and Seung-Gul Kang. Formal analysis: Jae Myeong Kang and Seung-Gul Kang. Funding acquisition: Seung-Gul Kang. Investigation: Jae Myeong Kang, Seo-Eun Cho, and Seung-Gul Kang. Methodology: Seon Tae Kim, Kee Hyung Park, and Seung-Gul Kang. Resources: Seon Tae Kim, Seo-Eun Cho, Gun Bae Lee, Kee Hyung Park, and Seung-Gul Kang. Supervision: Seung-Gul Kang.

\section{ORCID}

Jae Myeong Kang https://orcid.org/0000-0003-0803-9332 Seo-Eun Cho https://orcid.org/0000-0002-3991-2192

Gun Bae Lee https://orcid.org/0000-0002-1558-6038

Seong-Jin Cho https://orcid.org/0000-0002-8814-5807

Kee Hyung Park https://orcid.org/0000-0001-6847-6679

Seon Tae Kim https://orcid.org/0000-0002-2010-2843

Seung-Gul Kang https://orcid.org/0000-0003-4933-0433

\section{REFERENCES}

1. American Psychiatric Association. Diagnostic and statistical manual of mental disorders, fourth edition, text revision: DSM-IV-TR. 4th ed. Washington, DC:American Psychiatric Association; 2000 .

2. Berry RB. Sleep related breathing disorders: classification. In: Kryger M, Roth T, Dement WC, editors. Principles and practice of sleep medicine. Philadelphia:Elsevier;2017. p.10301040.e3.

3. Greenberg H, Lakticova V, Scharf SM. Obstructive sleep apnea: clinical features, evaluation, and principles of management. In: Kryger $M$, Roth T, Dement WC, editors. Principles and practice of sleep medicine. Philadelphia: Elsevier;2017. p.1110-1124.e6.

4. Schröder CM, O’Hara R. Depression and Obstructive Sleep Apnea (OSA). Ann Gen Psychiatry 2005;4:13.

5. Ohayon MM. The effects of breathing-related sleep disorders on mood disturbances in the general population. J Clin Psychiatry 2003;64:1195-1200; quiz, 1274-1276.

6. Wheaton AG, Perry GS, Chapman DP, Croft JB. Sleep disordered breathing and depression among U.S. adults: National Health and Nutrition Examination Survey, 2005-2008. Sleep 2012;35:461-467. 
7. Pillar G, Lavie P. Psychiatric symptoms in sleep apnea syndrome: effects of gender and respiratory disturbance index. Chest 1998;114:697-703.

8. Aikens JE, Mendelson WB. A matched comparison of MMPI responses in patients with primary snoring or obstructive sleep apnea. Sleep 1999;22:355-359.

9. Sforza E, de Saint Hilaire Z, Pelissolo A, Rochat T, Ibanez V. Personality, anxiety and mood traits in patients with sleep-related breathing disorders: effect of reduced daytime alertness. Sleep Med 2002;3:139-145.

10. Asghari A, Mohammadi F, Kamrava SK, Tavakoli S, Farhadi M. Severity of depression and anxiety in obstructive sleep apnea syndrome. Eur Arch Otorhinolaryngol 2012;269:2549-2553.

11. Kang JM, Cho SJ, Lee YJ, Kim JE, Shin SH, Park KH, et al. Comparison of psychiatric symptoms in patients with obstructive sleep apnea, simple snoring, and normal controls. Psychosom Med 2018;80:193-199.

12. Kaskie RE, Graziano B, Ferrarelli F. Schizophrenia and sleep disorders: links, risks, and management challenges. Nat SCi Sleep 2017;9:227-239.

13. Yang C, Winkelman JW. Clinical significance of sleep EEG abnormalities in chronic schizophrenia. Schizophr Res 2006; 82:251-260.

14. Ruhrmann S, Schultze-Lutter F, Salokangas RK, Heinimaa M, Linszen D, Dingemans $\mathrm{P}$, et al. Prediction of psychosis in adolescents and young adults at high risk: results from the prospective European prediction of psychosis study. Arch Gen Psychiatry 2010;67:241-251.

15. Jaussent I, Bouyer J, Ancelin ML, Akbaraly T, Pérès K, Ritchie $\mathrm{K}$, et al. Insomnia and daytime sleepiness are risk factors for depressive symptoms in the elderly. Sleep 2011;34:11031110.

16. Lauer CJ, Schreiber W, Holsboer F, Krieg JC. In quest of identifying vulnerability markers for psychiatric disorders by all-night polysomnography. Arch Gen Psychiatry 1995;52:145-153.

17. Schilling C, Schlipf M, Spietzack S, Rausch F, Eisenacher S, Englisch $\mathrm{S}$, et al. Fast sleep spindle reduction in schizophrenia and healthy first-degree relatives: association with impaired cognitive function and potential intermediate phenotype. Eur Arch Psychiatry Clin Neurosci 2017;267:213-224.

18. Wamsley EJ, Tucker MA, Shinn AK, Ono KE, McKinley SK, Ely $\mathrm{AV}$, et al. Reduced sleep spindles and spindle coherence in schizophrenia: mechanisms of impaired memory consolidation? Biol Psychiatry 2012;71:154-161.

19. Cheng P, Goldschmied J, Deldin P, Hoffmann R, Armitage R. The role of fast and slow EEG activity during sleep in males and females with major depressive disorder. Psychophysiology 2015; 52:1375-1381.

20. Duncan WC, Sarasso S, Ferrarelli F, Selter J, Riedner BA, Hejazi NS, et al. Concomitant BDNF and sleep slow wave changes indicate ketamine-induced plasticity in major depressive disorder. Int J Neuropsychopharmacol 2013;16:301311.
21. Chervin RD, Burns JW, Ruzicka DL. Electroencephalographic changes during respiratory cycles predict sleepiness in sleep apnea. Am J Respir Crit Care Med 2005; 171:652-658.

22. Alvarez D, Hornero R, Marcos J, Del Campo F, Lopez M. Spectral analysis of electroencephalogram and oximetric signals in obstructive sleep apnea diagnosis. Annu Int Conf IEEE Eng Med Biol Soc 2009;2009:400-403.

23. Lee YJ, Kim JW, Lee YJ, Jeong DU. Sleep EEG characteristics in young and elderly patients with obstructive sleep apnea syndrome. Psychiatry Investig 2016;13:217-221.

24. Lee HK, Park DH, Shin HS, Hong SC. Comparison of low resolution electromagnetic tomography imaging between subjects with mild and severe obstructive sleep apnea syndrome: a preliminary study. Psychiatry Investig 2008;5:45-51.

25. Appleton SL, Vakulin A, D'Rozario A, Vincent AD, Teare A, Martin SA, et al. Quantitative electroencephalography measures in rapid eye movement and nonrapid eye movement sleep are associated with apnea-hypopnea index and nocturnal hypoxemia in men. Sleep 2019;42:Zsz092.

26. Derogatis LR, Rickels K, Rock AF. The SCL-90 and the MMPI: a step in the validation of a new self-report scale. BrJ Psychiatry 1976; 128:280-289.

27. Kim JH, Kim K. The standardization study of symptom checklist-90-revision in Korea III. Ment Health Res 1984;2:278-311.

28. Iber C, American Academy of Sleep Medicine. The AASM manual for the scoring of sleep and associated events: rules, terminology and technical specifications. Westchester, IL:American Academy of Sleep Medicine,2007.

29. Dean DA 2nd, Goldberger AL, Mueller R, Kim M, Rueschman M, Mobley D, et al. Scaling up scientific discovery in sleep medicine: the national sleep research resource. Sleep 2016;39:1151-1164.

30. Mariani S, Tarokh L, Djonlagic I, Cade BE, Morrical MG, Yaffe $\mathrm{K}$, et al. Evaluation of an automated pipeline for large-scale EEG spectral analysis: the National Sleep Research Resource. Sleep Med 2018;47:126-136.

31. Buckelmüller J, Landolt HP, Stassen $\mathrm{HH}$, Achermann P. Trait-like individual differences in the human sleep electroencephalogram. Neuroscience 2006;138:351-356.

32. Chen Y, Wang Zy, Yuan G, Huang L. An overview of online based platforms for sharing and analyzing electrophysiology data from big data perspective. WIREs Data Min Knowl Discov 2017;7.

33. Nakamura $M$, Shibasaki $\mathrm{H}$. Elimination of EKG artifacts from EEG records: a new method of non-cephalic referential EEG recording. Electroencephalogr Clin Neurophysiol 1987;66: 89-92.

34. Choi IS, Ahn BJ, Jun BH, Chang CS, Sung JY. Psychological analysis with symptom check list-90-revision in patients with tinnitus. Korean J Otolaryngol-Head Neck Surg 2003;46:475480.

35. Bersagliere A, Achermann P. Slow oscillations in human non-rapid eye movement sleep electroencephalogram: ef- 
fects of increased sleep pressure. J Sleep Res 2010;19(1 Pt 2):228-237.

36. de Boer $M$, Nijdam MJ, Jongedijk RA, Bangel KA, Olff $M$, Hofman WF, et al. The spectral fingerprint of sleep problems in post-traumatic stress disorder. Sleep 2020;43:zsz269.

37. Neu D, Mairesse O, Verbanck P, Le Bon O. Slow wave sleep in the chronically fatigued: power spectra distribution patterns in chronic fatigue syndrome and primary insomnia. Clin Neurophysiol 2015;126:1926-1933.

38. Esser SK, Hill SL, Tononi G. Sleep homeostasis and cortical synchronization: I. Modeling the effects of synaptic strength on sleep slow waves. Sleep 2007;30:1617-1630.

39. Sharafkhaneh A, Giray N, Richardson P, Young T, Hirshkowitz M. Association of psychiatric disorders and sleep apnea in a large cohort. Sleep 2005;28:1405-1411.

40. Velasco-Rey MC, Sánchez-Muñoz M, Gutiérrez-López MI, Trujillo-Borrego A, Sánchez-Bonome L. Psychotic depression induced by Obstructive Sleep Apnoea Syndrome (OSAS): a case reported. Actas Esp Psiquiatr 2012;40:43-45.

41. Lombardi C, Rocchi R, Montagna P, Silani V, Parati G. Obstructive sleep apnea syndrome: a cause of acute delirium. J Clin Sleep Med 2009;5:569-570.

42. Baran AS, Richert AC. Obstructive sleep apnea and depression. CNS Spectr 2003;8:128-134.

43. Keshavan MS, Reynolds CF 3rd, Miewald MJ, Montrose DM, Sweeney JA, Vasko RC Jr, et al. Delta sleep deficits in schizophrenia: evidence from automated analyses of sleep data. Arch Gen Psychiatry 1998;55:443-448.

44. Oh SM, Lee YJ, Kim JW, Choi JW, Jeong DU. Preliminary study on quantitative sleep EEG characteristics in patients with schizophrenia. Psychiatry Investig 2017; 14:219-225.

45. Crowley K, Trinder J, Kim Y, Carrington M, Colrain IM. The effects of normal aging on sleep spindle and K-complex production. Clin Neurophysiol 2002;113:1615-1622.

46. Dubé J, Lafortune M, Bedetti C, Bouchard M, Gagnon JF, Doyon J, et al. Cortical thinning explains changes in sleep slow waves during adulthood. J Neurosci 2015;35:7795-7807.

47. Fogel S, Martin N, Lafortune M, Barakat M, Debas K, Laventure $\mathrm{S}$, et al. NREM sleep oscillations and brain plasticity in aging. Front Neurol 2012;3:176.

48. Helfrich RF, Mander BA, Jagust WJ, Knight RT, Walker MP. Old brains come uncoupled in sleep: slow wave-spindle synchrony, brain atrophy, and forgetting. Neuron 2018;97:221230.e4.
49. Mander BA, Rao V, Lu B, Saletin JM, Lindquist JR, AncoliIsrael S, et al. Prefrontal atrophy, disrupted NREM slow waves and impaired hippocampal-dependent memory in aging. Nat Neurosci 2013; 16:357-364.

50. Ehlers $\mathrm{CL}$, Kupfer DJ. Slow-wave sleep: do young adult men and women age differently? J Sleep Res 1997;6:211-215.

51. Mourtazaev MS, Kemp B, Zwinderman AH, Kamphuisen HA. Age and gender affect different characteristics of slow waves in the sleep EEG. Sleep 1995;18:557-564.

52. Reynolds CF 3rd, Monk TH, Hoch CC, Jennings JR, Buysse DJ, Houck PR, et al. Electroencephalographic sleep in the healthy "old old": a comparison with the "young old" in visually scored and automated measures. J Gerontol 1991;46:M39-M46.

53. Carrier J, Land S, Buysse DJ, Kupfer DJ, Monk TH. The effects of age and gender on sleep EEG power spectral density in the middle years of life (ages 20-60 years old). Psychophysiology 2001;38:232-242.

54. Bianchi MT, Williams KL, McKinney S, Ellenbogen JM. The subjective-objective mismatch in sleep perception among those with insomnia and sleep apnea. J Sleep Res 2013;22: 557-568.

55. Asghari A, Mohammadi F. Is Apnea-Hypopnea Index a proper measure for Obstructive Sleep Apnea severity? Med I Islam Repub Iran 2013;27:161-162.

56. Roure N, Gomez S, Mediano O, Duran J, Peña Mde L, Capote $\mathrm{F}$, et al. Daytime sleepiness and polysomnography in obstructive sleep apnea patients. Sleep Med 2008;9:727-731.

57. Kang JM, Kang SG, Cho SJ, Lee YJ, Lee HJ, Kim JE, et al. The quality of life of suspected obstructive sleep apnea patients is related to their subjective sleep quality rather than the apnea-hypopnea index. Sleep Breath 2017;21:369-375.

58. Puskás S, Kozák N, Sulina D, Csiba L, Magyar MT. Quantitative EEG in obstructive sleep apnea syndrome: a review of the literature. Rev Neurosci 2017;28:265-270.

59. Svanborg E, Guilleminault C. EEG frequency changes during sleep apneas. Sleep 1996;19:248-254.

60. Morisson F, Lavigne G, Petit D, Nielsen T, Malo J, Montplaisir J. Spectral analysis of wakefulness and REM sleep EEG in patients with sleep apnoea syndrome. Eur Respir J 1998;11: 1135-1140.

61. Walsleben JA, O'Malley EB, Bonnet K, Norman RG, Rapoport DM. The utility of topographic EEG mapping in obstructive sleep apnea syndrome. Sleep 1993;16(8 Suppl):S76-S78. 